\title{
POSTER
}

\section{Protocole de prise en charge en chirurgie orale d'un patient traité par sunitinib : Revue de la littérature et présentation de cas cliniques}

\section{Fongaufier $\mathrm{C}^{1}$, Barthélémy $\mathrm{P}^{2}$, Aubertin-Kirch $\mathrm{G}^{3}$, Gros $\mathrm{Cl}^{1-4}$, Bridonneau $\mathrm{T}^{1}$, Bornert $\mathrm{F}^{1}$}

1 - Unité de Pathologie-Chirurgie buccale, UF 8601, Pôle de Médecine et de Chirurgie bucco-dentaires, Hôpital Civil, Hôpitaux Universitaires de Strasbourg 67000

2 - Département d'Oncologie médicale-Hématologie, Hôpital Civil, Hôpitaux Universitaires de Strasbourg

3 - Département de Pharmacologie, Faculté de Médecine de Strasbourg

4 - Unité de Radiologie, UF 8609, Pôle de Médecine et de Chirurgie bucco-dentaires, Hôpital Civil, Hôpitaux Universitaires de Strasbourg

\section{christelle.fongaufier@gmail.com}

L'avènement des thérapies ciblées au cours de la dernière décennie représente une avancée majeure dans la prise en charge des cancers.

Malgré une amélioration de la survie des patients, les thérapies ciblées présentent de nombreuses toxicités sur la sphère digestive et en particulier la sphère orale.

Le sunitinib est un inhibiteur de tyrosine kinase (TKI) de la famille des antiangiogéniques qui cible et inhibe l'activité kinase du domaine intracellulaire de différents récepteurs transmembranaires et en particulier le VEGFR (vascular endothelial growth factor receptor), et le PDGFR (Plateletderived growth factor receptor) (1).

Le sunitinib est le chef de file de la famille des TKI antiangiogéniques qui comprend encore le pazopanib, sorafénib, cabozantinib ou encore l'axitinib. Le sunitinib présente une AMM dans 3 indications : les cancers du rein avancés ou métastatiques, les tumeurs stromales gastrointestinales (GIST) de l'adulte métastatiques et les tumeurs neuroendocrines du pancréas non résécables ou métastatiques.

Les effets du sunitinib sur la sphère buccale ont rapidement été mis en avant dès les premiers essais cliniques en particulier dans les cancers du rein : dysgueusie, xérostomie, gingivorragies, stomatite. Après sa mise sur le marché, des cas d'ostéonécrose mandibulaire (ONM) dans les suites d'un geste bucco-dentaire ont été rapportés en absence ou en présence de thérapies ciblant l'os comme les bisphosphonates ou plus récemment le dénosumab prescrites en raison de métastases osseuses (2).

À ce jour, le rôle des TKI antiangiogéniques dans la survenue des ONM est reconnu bien que le mécanisme d'action reste obscur. L'indication des bisphosphonates et/ou du dénosumab en association à un TKI antiangiogénique dans la prise en charge des métastases osseuses notamment des cancers du rein reste débattu. En absence de données robustes la prise en 
charge actuelle n'est pas standardisée. De nombreuses études sont en cours afin d'évaluer le risque réel d'ONM en cas d'association antiangiogéniques et thérapies ciblant l'os.

L'élargissement des indications du sunitinib et le retentissement sur l'état général des malades de l'ostéonécrose mandibulaire nous mènent à nous poser la question d'une conduite à tenir protocolisée face à l'indication d'une chirurgie buccale et en particulier une extraction dentaire chez les patients sous sunitinib ou autre antiangiogénique.

Dans les cas où l'extraction est inévitable, il paraît nécessaire d'envisager en étroite collaboration avec l'oncologue une fenêtre thérapeutique et /ou d'une diminution de la posologie. La décision doit être prise en fonction de la réponse tumorale au traitement, du pronostic de la maladie, de l'altération de la qualité de vie secondaire aux pathologies bucco-dentaires, ainsi que de l'existence d'autres effets indésirables. II faut également tenir compte de la pharmacodynamie de la molécule (3).

Cette discussion sera illustrée par cinq cas de patients sous sunitinib pris en charge conjointement par les services de chirurgie orale et d'oncologie médicale du CHRU de Strasbourg.

L'objectif de l'exposé est de proposer une stratégie thérapeutique oncologique et bucco-dentaire pouvant répondre à la majorité des situations cliniques des patients traités par sunitinib, et d'envisager par la suite un élargissement de cette conduite à tenir à d'autres molécules ayant des propriétés biologiques similaires (sorafénib, bévacizumab, axitinib...).

\section{Références}

(1) Aparicio-Gallego G. et al. New insights into molecular mechanisms of Sunitinib-associated side effects. Mol Cancer Ther. $2011 ; 10$ (12) : 2215-23.

(2) Koch F et al. Osteonecrosis of the jaw related to sunitinib. Oral Maxillofac Surg. 2011 ; 15 : 63-66.

(3) Houk B. et al. Relationship between exposure to sunitinib and efficacy and tolerability endpoints in patients with cancer : results of a pharmacokinetic/pharmacodynamic meta-analysis. Cancer Chemother Pharmacol. 2010 ; 66 : 357-37. 